\title{
The Suicidal Self in Cyberspace: Discursive Constructions of Identity in a Pro-Recovery Suicide Forum
}

\author{
Mike Alvarez* \\ Department of Communication, University of New Hampshire, Durham, NH, United States
}

The proliferation of suicide-related content online has led to widespread fears that suicidal persons are at elevated risk in our networked society. Though much research has been done on the benefits and harms of digital technologies, few studies have attended to the deep discursive meanings co-created by suicidal users. The present study attends to meanings about identity interactionally created by members of SuicideForum.com (SF), a pro-recovery website. Methodologically, I collected a purposive sample of 2,119 posts across 131 threads, which I then examined using cultural discourse analysis, tracking discursive hubs like "suicide" and "suicidal" to arrive at corresponding radiants of meaning. Findings reveal two sets of discursive themes: one set speaks to problematic identities, the other to a self emancipated from suicidality. The suicidal self is expressed in discourse as the product of a fractured identity, marked by schism between an authentic "inner self" and

OPEN ACCESS

Edited by:

Yukari Seko,

Ryerson University, Canada

Reviewed by:

Karolina Krysinska,

The University of Melbourne, Australia

Zhenyi Li,

Royal Roads University, Canada

*Correspondence: Mike Alvarez

mike.alvarez@unh.edu

Specialty section:

This article was submitted to Health Communication,

a section of the journal Frontiers in Communication

Received: 02 March 2021 Accepted: 18 May 2021

Published: 31 May 2021

Citation: Alvarez M (2021) The Suicidal Self in Cyberspace: Discursive Constructions of Identity in a Pro-Recovery

Suicide Forum.

Front. Commun. 6:674893.

doi: $10.3389 /$ fcomm.2021.674893 a socially aligned but inauthentic "outer self." However, resolution of fractured identities depends not on harmonious fusion but on reconciliation. Moreover, staying alive-continuing to be-is contingent upon recognizing that every life, including one's own, has value, finding purpose and meaning, and tending to others' well-being. The analysis uncovers discrepancies between SF users' folk understanding of the suicidal self and that espoused by dominant (i.e., biopsychiatric) models. Their implications for clinical and therapeutic practice are discussed.

Keywords: suicide, internet, digital media, cybersuicide, cultural discourse analysis, ethnography of communication

\section{INTRODUCTION}

The advent of Web 2.0, which has facilitated the creation of user-generated content online and enabled new forms of sociality among persons with discreditable stigmas (Goffman, 1963), has been met with both fears and hopes by scholars studying mental health and suicide. On one hand, there are widespread concerns that suicidal persons are at elevated risk in our networked society given the profusion of triggering content circulating on the Web. On the other hand, the presence of online communities that tend to their members' affective needs (Giles and Newbold, 2013), and mental health professionals' use of information and communication technologies (ICTs) to identify and extend help to people in crises (Quinnett and Baker, 2009), have allayed some of these concerns. The popular and scholarly discourses on cybersuicide (Sher and Vilens, 2009) have been structured by what Alvarez (2020) calls the benefits-harm paradigm, which is unsurprising given that the mental health disciplines, which have a large claim on the study of cybersuicide, are committed to maximizing well-being and reducing risk. 
The harmful content, contexts, and uses identified in the literature are manifold. The most culturally salient is suicide due to cyberbullying, or bullycide (Recupero, 2012), which taps into fears surrounding digital and mobile media's capacity to extend the temporal and spatial reach of cruel behavior. Less salient to the popular imagination are asynchronous pro-suicide message boards, where users' motives for suicide are deemed valid (Westerlund, 2013), self-destructive behaviors are encouraged (Ikunaga et al., 2013), and voluntary death is framed as emancipatory, a legitimate release from suffering (Baume et al., 1997). Digital media's capacity for dissemination, and the ease with which digital information can be accessed, has also given rise to concerns about the proliferation of suicide means and methods online (Chang et al., 2011; Gunnell et al., 2015). These concerns are stoked by the presence of online suicide manuals, which provide step-by-step instructions on committing suicide (Westerlund, 2011), and online message boards where tips and tricks for enacting self-harm are freely shared (Westerlund, 2013).

Digital media have ushered new forms of visibility and sociality among suicidal persons, outside of therapeutic contexts typically presided by professionals. In addition to the harms already mentioned, we may also add net suicide pacts, in which two or more individuals who meet online agree on a time and place to end their lives together (Rajagopal, 2004). Unlike traditional suicide pacts, which form between intimates in real life in response to threats to the continuation of a relationship (such as a spouse or partner's diagnosis with a terminal illness), remediated suicide pacts form between strangers online, who in death are said to achieve the closeness they perceived to be missing in life (Ozawa-de Silva, 2008, Ozawa-de Silva, 2010; Seko, 2008). Far less common is deathcasting, in which an individual broadcasts his or her suicide to a live virtual audience, sometimes due to pressure from anonymous viewers who goad the user with incendiary messages (Stamenkovic, 2011). Seko (2018) links this "emergent visibility of the "suicidal" self" (p. 173) in liquid modernity to the desire to witness and to be seen and recognized.

Of course, for every destructive potential of the internet, ${ }^{1}$ there is a corresponding constructive potential. In the realm of suicide prevention and intervention, these include professional websites that disseminate empirically validated information, such as risk factors, warning signs, and treatment options (Quinnett and Baker, 2009); the online delivery of various treatment modalities, such as e-therapy and telemedicine, to at-risk populations (Mewton and Andrew, 2015); and gatekeeper training programs for professionals likely to come into contact with suicidal persons (Aboujaoude and Starcevic, 2015). A number of

${ }^{1}$ My spelling of "internet" with a lower-case " $i$ " is deliberate. I share Baym and Markham's (2009) sentiment that using the upper-case "I" "suggests that 'internet' is a proper noun and implies that it is either a being [...] or that it is a specific place $[\ldots]$ granting the internet agency and power that are better granted to those who develop and use it" (p. vii). professional websites contain message boards where users can solicit advice from other users, or from clinicians who serve as moderators. Of course, pro-recovery sites are not run exclusively by professional organizations. There exist, for example, social networking sites (SNS) run by suicidal persons and their loved ones (Biddle et al., 2016), support groups within existing SNS like Facebook (Mars et al., 2015), and blogs and blog circuits that narrativize the struggle with suicidality (Singaravelu et al., 2015).

Aside from classifying the different types of pro-recovery content on the Web, there is also interest in cataloguing the actual benefits derived by users from participating in interactive contexts. Such benefits might include, for example, a sense of community, empathetic understanding, and coping mechanisms for managing stressful life events (Baker and Fortune, 2008). Since one of the hallmarks of suicidality is ambivalence (Joiner, 2005), participation in supportive contexts can be life-saving, with highly vulnerable users renegotiating their lease in life by halting or delaying suicide plans as a result of contact with empathetic others (Ekman and Söderberg, 2009). Finding someone who shares one's stigma can replace feelings of abnormality with that of shared humanity, and ultimately, swing the pendulum towards life and away from death.

Though it remains useful, the benefits/harm paradigm, which taps into utopian hopes and dystopian fears about the internet, can sometimes efface the syntopian aspects of digital communication-that is, its capacity for meaning generation (Katz and Rice, 2002). In many studies of cybersuicide, the unit of analysis is the individual, who is envisioned as suffering from an underlying pathology (Seko, 2018), and technology is viewed as a double-edged tool that can exacerbate or ameliorate said condition-a view not unlike the transmission or effects models that dominated the early decades of mass media studies. Moroever, the internet is often conceived as monolithic, rigidly deterministic of human behavior, and users are passive recipients of harmful or beneficial content, instead of agents who use the platform's affordances to create meaning. Because the emphasis is on identifying threats and opportunities, promises as well as pitfalls, and because the unit of analysis is the individual rather than group, (sub)culture, or community, the deep discursive meanings active in participants' messages online are sometimes overlooked.

The present study is a modest attempt to arrive at discursive meanings about identity and personhood interactionally created by users of a pro-recovery suicide forum. I respond to Giles and Newbold's (2013) call to treat online mental health forums as speech communities or communities of practice, and mental health conditions as subcultural identities. Like Thompson (2012) in their study of 17 disorder communities online, I pay attention to the discursive moves participants rely on to signal themselves as members. Lastly, instead of treating the internet as a mere tool, I adopt Markham's (1998) view of the internet as a heterogeneous space in which discursive communities can jointly craft meanings about their communicative worlds and their personal and social identities. 


\section{THEORIES AND METHODS}

For this study, I adopt Carbaugh's (2012) four-part, communication definition of culture as 1) an expressive system, 2) of symbols and symbolic forms, 3) that are deeply meaningful to participants in place, 4) and are transmitted by members across time. I then heed Hecht's (2010) call to extend culture to entitities not previously considered as such-by viewing "suicidal" individuals as a cultural rather than a diagnostic category, and users of a pro-recovery suicide forum as members of a speech community, defined by Hymes (1972) as a group of people whose discursive practices and strategies of meaning making underlie common values and beliefs.

Methodologically, I enlist cultural discourse analysis (CuDA) (Carbaugh and Hastings, 1992; Carbaugh, 2007, Carbaugh, 2017; Scollo, 2011) because it is a rich tool for theorizing communication generally and communicative practices specifically; for describing expressive activities in great depth; and for interpreting the meaning of discursive practices to those who engage in them, in their own terms, thereby honoring Geertz (1973) commitment to "thick description." CuDA has a rich theoretical lineage that includes the ethnography of communication (Hymes, 1972) and theories of speech codes (Philipsen, 1987) and cultural communication (Philipsen, 1997), which see "membering" as the communal function of social interaction. In the three decades since its inception, CuDA has been productively applied to various communicative practices and discursive communities, including users of websites and online platforms (see Scollo and Milburn, 2019, which collects the writings of $42 \mathrm{CuDA}$ practitioners worldwide).

$\mathrm{CuDA}$ has two key assumptions. The first, known as the "axiom of particularity" (Carbaugh et al., 1997, p. 3-4), is that communication is used, valued, and conceived of in locally distinctive ways. In other words, communication is particular to places and varies from one context to the next. The second, known as the "axiom of actuality" (p. 4), is that in any given place, a system of communicative practices already exists, and through those practices, members are able to give form, order, and meaning to (i.e., actualize) their social lives. Such practices are infused with deeply meaningful messages called cultural discourses, so that when participants speak, they are not only saying something about the topic at hand; they are making metacultural commentaries about how to act, how to feel, how to relate to others, how to be, and how to inhabit the world. Carbaugh (2007) calls these the five radiants of meaning (p. 174) ${ }^{2}$-acting, feeling, relating, being, and dwelling, in that order which are invoked each time discursive hubs (ibid.) are used.

Whereas radiants are the implicit meanings, hubs are the explicit units of analysis. They may take the form of words, phrases, gestures, images, symbols, and symbolic forms that are potent to a group, (sub)culture, or community, their

\footnotetext{
${ }^{2}$ In more recent writings, Carbaugh and other practitioners of $\mathrm{CuDA}$ have added a sixth radiant: timing (see, for example, Scollo and Milburn, 2019), though one can argue that each of the five original radiants has a temporal dimension that is implicit or explicit in discourse.
}

meaningfulness apparent in their frequency of use, emphatic usage, mutual intelligibility, and accessibility to participants. Hubs and radiants are inseparable; a single hub can invoke multiple radiants at once, and hubs can work in isolation or in tandem to activate meaning; "a hub need not necessarily be explicitly affiliated with one and only one radiant of meaning" (Carbaugh, 2019, p. 319). To give one example: in an online thread where self-injurers share their reasons for self-harming (Alvarez, 2020), tracking the word "self-harm"-a discursive hub of action-reveals participant beliefs about self-discipline and mastery (radiant of acting), emotion management (feeling), troubled relationships (relating), and self-worth, or lack thereof (being). However, not all hubs/radiants are relevant or salient in every communicative scene - meanings about place ( $d$ welling) were not invoked in the example mentioned-and for analytical purposes, the researcher can track one radiant at a time.

The present study is drawn from a much larger, ethnographic study of a pro-recovery suicide website called SuicideForum.com (SF hereafter). ${ }^{3} \mathrm{SF}$ is one of the largest websites dedicated to discussing suicide, and since its creation in 2005, it has amassed over 120,000 threads, 1.4 million posts, and 27,000 registered users worldwide. SF brands itself as a "peer to peer community support forum and chatroom for people in need," and it abides by a "Do no harm, promote no harm principle" (www.suicideforum. com/about-sf/). What this means is that while participants are free to discuss their struggles with suicide (past or present), they are prohibited from posting specific suicide plans or timelines, encouraging others' plans, soliciting suicide partners, sharing suicide methods, and discussing past attempts in minute detail, all of which are deemed triggering to other users. Moderators redact messages with triggering content, and users who commit infractions are issued sanctions in the form of points ranging from zero to twelve; accumulating twelve points results in immediate account termination. As mentioned, the site is expressly pro-recovery, defined by members as an active and earnest commitment to improving mental health (one's own and that of others) by providing informal online support in conjuction with formal treatment received elsewhere offline.

Besides the forums, the site provides reference materials on various mental health conditions, as well as numbers for crisis hotlines and links to crisis websites for seventy-nine countries. Though membership is not required to access much of the site's content, registration (which is free and requires no personally identifying information) is necessary for posting and participating in threads. Communication in SF is primarily in English. The site is staffed by volunteers and its operations are supported entirely by donations, which cover server costs, software updates, security and licensing. SF is not run or moderated by any particular organization, but by former members who now serve as staff or administrators. These individuals volunteer their time to perform various tasks, such as monitoring threads for objectionable content; responding to member queries, reports, and complaints; imposing sanctions for offenses; and maintaining site functionality.

${ }^{3}$ For preliminary findings, see Alvarez's section in Flanigan and Alvarez, 2019. 
Prior to the investigation, I spent seven months informally browsing SF to familiarize myself with the site's layout, technical affordances, diversity of content, rules of conduct, and communicative norms. This was followed by thirty weeks of non-participant observation, where I utilized Hymes's (1972) descriptive theory to tease apart the components of the communication scene in question, which are captured by the mnemonic SPEAKING, ${ }^{4}$ in order to understand how the website's discursive architecture shapes the meanings created therein. Given the huge volume of data on SF, I then collected and analyzed a purposive sample of messages within a restricted time frame, rather than set an a priori number of posts, which goes against CuDA's spirit of discovery and immersion. During the 30 week period, I collected 2,119 posts across 131 threads, making sure posts were drawn from every major section of the forums. ${ }^{5}$ The posts amount to 683 pages of transcripts. In order to preserve the message structure of threads, no data scraping software were used during the collection process. All consulted threads were printed to facilitate hand coding, which I conducted by myself.

Using cultural discourse analysis, I systematically tracked the usage of "suicide," a discursive hub of action, and "suicidal," a discursive hub of emotion (as in feeling suicidal) as well as personhood (as in being suicidal), in participants' online discourse to arrive at corresponding radiants of meaning. I tracked each of the five aforementioned radiants one by one, formulating one research question for each. For each radiant, I conducted multiple rounds of coding to maximize completeness and accuracy. In the initial coding stage, I generated as many categories as possible based on recurring patterns in the transcripts. I then reviewed the transcripts to verify said categories, check for discrepancies, and identify additional categories previously missed. In the next stage, I grouped together related categories to identify overarching discursive themes and sub-themes.

In the course of the analysis, two major sets of themes emerged: 1) discursive pathways to suicide, or problematic ways of being, relating, acting, feeling, and dwelling; and 2) discourses of positive treatment and recovery, or emancipatory ways of being, relating, acting, feeling, and dwelling. Rather than position these themes as contrastive sets, it is perhaps more productive to view them as two parts of a discursive continuum that expresses pathways into and out of suicide. In the section that follows, I present meanings about personhood (being) that are invoked in SF users' online discourse by responding to the following research question: What models of personhood are presumed when participants discuss precipitants to and recovery from suicide? These interpretive accounts take the form of cultural premises (italicized

\footnotetext{
${ }^{4}$ The components are: Setting (S), participants (P), ends/goals (E), act topics/act sequence (A), key/emotional pitch (K), instruments/channels (I), norms of interaction/interpretation $(\mathrm{N})$, and genres of communication $(\mathrm{G})$.

${ }^{5}$ During the study period, there were seven major sections: New Members, Suicidal Thoughts and Feelings, Road to Recovery, Let It All Out, Support and Advice, You Are Not Alone, The Gathering.
}

throughout), which Carbaugh (2007), Carbaugh (2017) defines as abstract formulations that capture participants' taken-forgranted knowledge and beliefs about the way things are and the way things ought to be. I support each cultural premise with brief excerpts from forum threads.

Before presenting findings, a few words on ethics are in order. Neither intervention nor interaction with SF users took place, and analysis was limited to extant data on publicly accessible threads with heavy traffic. Furthermore, individual posts are not linked to personally identifying information, and site rules prohibit members from using their real-life photo as their avatar, from using their real name as their handle, and from reusing handles associated with accounts on other websites and platforms. In short, transcripts are already anonymized, and I encountered no evidence to the contrary throughout data collection and analysis. A related concern pertains to researchers' legal and ethical responsibilities upon encountering distressing information online, where there is imminent threat to the physical safety of groups or individuals (Stern, 2003). However, given SF's clear enforcement of its "Do no harm, promote no harm" principle, I did not encounter posts that warranted intervention on my part.

\section{FINDINGS}

In what follows, I present two sets of discursive themes that radiate from SuicideForum users' online discourse. As it will become evident, each set contains multiple variations of a central organizing theme. In the first section, I begin by providing context via an overview of SF users' clinical and sociodemographic characteristics. I then explore users' ontological view of suicidality as a product of fractured identity, which expresses itself in discourse in a variety of ways-for instance, as schism between an authentic inner self and an artificial outer self, between real and ideal(ized) selves, and temporally, between past, present, and future selves. In the second section, I discuss the ideal selves that are emergent in SF discourse. From SF users' discursive point of view, the person liberated from suicidality is greater than his or her biological makeup; has a sense of purpose; is cognizant of his/her inner fortitude; and is embedded in a web of meaningful relations-among other defining qualities. This model person has implications for how SF users reconcile the split self that contributes to suffering in the first place.

\section{The Discursive Ontology of Suicidality}

Clinically, registered users of SuicideForum.com (SF) wrestle with various mental health conditions, including substance abuse, anxiety, disordered eating, post-traumatic stress, attention deficit and hyperactivity, borderline personality, schizophrenia and other psychoses, and of course, depression. This partial list underscores the notion that suicide is not isomorphic with any one condition, but instead cuts across them. Users also vary in their attribution of suicidality to endogenous (arising from within) or exogenous (external to oneself) forces. Some espouse endogenous explanations (e.g., "please remember this is an illness"), while others embrace exogenous explanations ("You 
probably have good reasons for being this way, like some time in your life where a lot of bad things happened"). Participants also complain of various physical ailments ranging from acute to chronic, such as diabetes, tuberculosis, chronic pain, and multiple sclerosis.

Socio-demographically, it is not possible to ascertain the racial and ethnic make-up of users through non-participant observation, especially when the subject of race-ethnicity rarely enters the discourse. It is also not possible to ascertain class distribution, though some users report financial struggles such as loss of employment, underemployment, and concerns over making timely payments on bills and mortgages. However, it is apparent that SF embraces a spectrum of gender and sexual identities, with users presenting as male, female, or transgender, and as lesbian, gay, bisexual, queer, intersex, or asexual. Moreover, forum participants are also diverse in terms of age, with 13 years as the minimum age requirement for registration. The site's age inclusivity is reflected in the Forums' discursive architecture, with the "Generation Gap" section divided into "Adolescence and Young Adult," "Mid-Life," and "Late Life/Seniors." Lastly, the site is open to practitioners of all faiths and non-practitioners alike, provided that users do not impose their religious beliefs-or non-adherence to a religious doctrine-onto others.

As this brief portrait illustrates, members of the SF community come from different walks of life. Despite their differences, however, tracking their usage of "suicide," "suicidal," and other discursive hubs of interest, reveals an ontological view of suicidality as the product of fractured identity.

The fragmentation expressed in discourse presumes an "inner" self and an "outer" self that are profoundly disconnected from one another. In SF members' forum posts, the metaphor of the ocean is frequently invoked to illustrate this schism. Discussing their avatars, two members write:

The ocean to me is a reflection of my inner self, looks can be deceptive, sometimes it's peaceful and quiet and other times in turmoil, bubble and trouble, it holds beauty within but dangers can also lurk deep inside

I love the ocean, its so powerful, and the way the waves are crashing against the rock reminds me of the inner turmoil i sometimes feel.

The ocean is discursively elaborated through such qualities as "peaceful" and "quiet" on the surface, but full of "danger" and "turmoil" underneath its calm veneer. So potent is this inner turmoil that it can cause waves to swell and crash against rocks. Such is the suicidal person, who might appear tranquil on the outside but contain so much depth of feeling that they threaten to implode.

In discourse, members characterize the outer self that they project to the world as artificial or fake, and the inner self, which is hidden from view, as real and authentic. For instance, one user writes: "I feel like I have to put on this metaphorical outfit and wear a fake smile just to fit in with my classmates and my family." Referring to their avatar, another user writes: "I hide behind a mask and my avatar represents my hidden struggle." Both examples involve donning a performative exterior that is profoundly misaligned with an invisible interior. Of course, one can argue that everyone experiences some discrepancy between public and private, front stage and back stage (Goffman, 1959), and in some speech communities, bifurcation of the self is not only expected but natural (Geertz, 1973; Kotani, 2002). In SF members' discourse, however, the discrepancy is not only expressed as immense but problematic, too, causing one to "suffer in silence." This may be erected on the popular American notion that the inner should be aligned with the outer for one to have an "authentic self" (Carbaugh, 2005). But in the case of SF users, the inner self must be kept secret because it contains that which society deems unacceptable. Wrote one user: "People tend to make public the things that society approves of, and then hide all the rest."

The fragmentation of self is also expressed in discourse temporally, along the axis of time. One variant is a discrepancy between past and present selves, between the person one formerly was, and the person one currently is. When asked why s/he attempted suicide, one member responds: "I had recently quit the track and field and running was my life for years before that so I felt completely lost." For this user, being a runner constituted a vital part of their identity; to cease being a runner was to risk ceasing to be. In response to the same question, another member writes: "I was a new father and couldn't handle it." For this user, being suddenly thrust into a new role - and a new self - overwhelmed any attempt at identity alignment. Another variant expresses itself as a discrepancy between present and future selves. One overweight member of the SF community wants so very badly to become thin that she "gets angry at myself for eating," and falling short of her punitive self-discipline provokes suicidal crises.

In both temporal variants, there is once again a chasm between a socially aligned outer self, and an ideal(ized) inner self, and this chasm is a source of agony for SF users. Misalignment and incongruence between split selves can eventuate in feelings of worthlessness: "I felt worthless," "pathetic and socially inept," "I've done nothing good in life to be treated well." The deep dissatisfaction with the self that we have seen thus far is especially palpable in the online communication of members who are unhappy with their sex at birth. For instance, two users write, "I didn't want to be a male," and "I am a woman and I HATE it!" A third user adds: "Being in a male body for 17 years is far too long [...] more than a decade of not being the woman you identify and you truly are, can be detrimental!" In these examples, one's true gender identity - the authentic inner self - is imprisoned in an incompatible, yet socially prescribed, biological body.

To summarize, the ontology of suicidality from SF members' discursive point of view can be captured by this larger cultural premise: There is a self, and when fragmented or bifurcated into incongruent parts - a problematic inner self and a socially aligned outer self - great anguished is produced, which can manifest in thoughts and feelings of suicide. But as we will see shortly, the discourses of SF users are also rich with meaningful prescriptions for emancipating the self from the call of self-destruction.

\section{A Discourse for Ideal and Emergent Selves}

In response to a user's complaint that $\mathrm{s} / \mathrm{he}$ does not meet objective standards of normalcy ("[n]ormal is the opposite of 
me. . . everything I am not"), one user, quoting the scientist Neils Bohr, writes: "[H]ow problematical such concepts as "objective" and "subjective" are." To this another user adds: "Maybe you should define what normal is and give you a better idea of what you want to be." From this quick snapshot, it becomes apparent that participants respectfully correct one another's selfdefeating evaluations without discrediting the underlying feelings. Upon closer inspection, such correctives also tap into the ideal self that SF users construct in discourse notions of self that challenge objective standards of what it means to be human.

The ideal self that is discursively constructed by SF users is greater than his/her biological makeup. Beyond the fulfillment of bodily needs and cravings, this person also takes into consideration matters of the heart, so to speak: "I learned that life is so much more than physical wants and needs and desires. My heart was broken and needed healing too." Furthermore, this person has a sense of purpose, which allows him/her to live life meaningfully: "You have a purpose in life and you are here for a reason." The notion that life and death are not merely biological phenomena, but existential ones too, is apparent in one member's reservations about taking medication: "[M]eds may take away being suicidal but will still leave me with an empty existence, I would go through life just existing with no purpose. I don't want that." Psychotropic medication may or may not ameliorate suicidality, but it will certainly not give one purpose. It may keep one from falling off the proverbial cliff, but in order to pull oneself up onto the ledge, a sense of purpose is needed.

To resist suicide at every turn requires "willpower," but without purpose, such willpower is limited. Wrote one user: "Sure, willpower will get us so far, but everyone runs out of willpower at one point or another. What truly spurs us on in the moments that our willpower is lacking, is having a deeper meaning and purpose to our lives." A sense of purpose can come from many sources including, but not limited to, one's passions and aspirations for the future: "[W] hat is it that you care about, what do you live to do, what are your passions, what would truly make you happy in this world, what do you want to achieve in this lifetime?" One can also derive purpose from one's suffering, which users deem a natural part of existence. This does not mean seeking out suffering to imbue one's life with meaning; in the words of one user, "that would be false martyrdom."

A person with purpose has a future to look forward to. Phrased another way, purpose can be located in the future. On their own, the suicidal person cannot envision a future for themselves ("no future prospects"), or sees only greater misfortune in the horizon ("I just have a life of failure ahead of me"). Within the context of SF, such negative evaluations are respectfully corrected by other users by locating purpose in a future that has yet to come to fruition: "You've got so much left in life to accomplish"; "You don't realize what great things await you." Such comments resonate with Hecht's (2014) argument that the suicidal person owes it to his or her future self to continue living. To end one's life is to tragically deny one's future self the myriad opportunities for happiness and self-fulfillment that await.
SF users agree in discourse that in order to lead better lives, they need to change as persons. The desire for metamorphosis into a "freer" person is palpable in users' messages to one another. This yearning is expressed by one user's identification with a butterfly (their avatar of choice), which symbolizes "that I am in a bad place but trying to stay strong through these bad times and that I am hoping that things change just like the butterfly did from the caterpillar." A similar metaphor is enlisted in another post, with the user identifying with "a bird soaring to symbolize flying away from bad things which I want to do but don't know if strong enough yet." In both examples, old and new selves are differentiated spatially. The old self is earthbound and trapped, whereas the new and idealized self is free to roam the skies.

For change to happen, one must recognize the wellspring of strength within. According to one user, deep inside every person resides "a greater strength...that none of us really know that we have until we are forced to use it." It is easier to intuit this strength when it is exhibited by others, as in the case of a user who wishes to be like a lion: "bold, strong, proud, qualities i wish i possessed, while still being caring for members of their pride." But recognition of strength in oneself is vital for transformation to occur. For members of the SF community, perhaps the best proof of one's strength lies in enduring, and surviving, suicide. In response to a user who feels like a "punching bag," the passive recipient of life's relentless onslaughts, another user responds: "just thinking punching bags are designed to withstand even what the best fighters can throw at them, and when they are beat the punching bag is still intact." In short, to endure adversity with one's life intact is to exhibit resilience, which is evidence that one possesses strength.

Previously, I mentioned that SF users discursively conceive of the suicidal self as fragmented or bifurcated. However, recovery is not contingent upon the disavowal of negative aspects of the self, or the "naïve" reconstitution of splintered parts into a seamless whole. Rather, it is the reconciliation of contradictory parts-not in perfect harmony, but in bearable tension-from which the possibility of self-transformation and recovery springs. In this regard, the myth of Persephone is particularly resonant to users. According to Philipsen (1987), when invoked in discourse, myths not only bind together the imagination of community members; they also provide prescriptions for meaningful thought and action.

Persephone is the Greek goddess of the seasons who, upon eating six pomegranate seeds in the Underworld, is forced to spend six months of every year with Hades, after which she returns to the heavens and the mortal realm. Persephone's ascent from the Underworld marks the arrival of spring and then summer, and upon her inevitable descent, fall and winter follow. Observe what one user, who has chosen to base their avatar and handle on Persephone, has to offer:

My avatar is Persephone (or Proserpina), by Rossetti. Persephone is my primary online name. She is the goddess of both Spring and the Underworld in Greek mythology. I view her as symbolizing the cycle of life 
and death, change and transformation. She also symbolizes being able to travel between worlds, between different states of consciousness, and between the normal world of the living and world of magic and mystery.

In the extract, we can see the invocation of agonistic terms: "Spring" and "Underworld," "life" and "death," "normal" and "magic and mystery." The mythic figure of Persephone encompasses these agons, suggesting the transformative potential of navigating the manifold contradictions of the self. When one braves into the Underworld of one's soul, one emerges transformed. Such is the descent into and rise from the depths of suicidality.

The ideal person changes for the better, not for the worse. S/he remains a good person in spite or because of adversity, "a good person to the core, even when faced by dark times." In discourse, the transformed person is also more "carefree"- "without a care for what others think of them," and "not giving a single damn at all about anything." Of course, being "carefree" does not mean forsaking all responsibilities, for SF users believe that they have a social obligation to try and "make other people happy," or at least not contribute to others' unhappiness. As examples throughout this article have shown, their supportive orientation is apparent in the support they endeavor to provide others, within and beyond the discursive space of SF. Being "carefree" is also not synonymous with the pursuit and attainment of unadulterated happiness. From SF users' discursive point of view, true happiness is pure fantasy; "the best one can hope for is to be mostly happy."

The emancipated self is embedded in a web of meaningful relations. The first-person plural "we" in particular is a very powerful discursive hub of personhood, and its prolific use by SF members (e.g., "You're not suffering in silence anymore, man. We've got you"), even when referring to one's own personal views or feelings ("We care for you"; "We like you"), suggests a strong sense of affiliation that emerges as a result of participation in the site. The discursive move away from singularity towards relationality is accompanied by recognition that suicidal individuals do not have a monopoly on suffering: "Everyone suffers in life. That's not to say that life is bad for everyone, but rather everyone has some time of hardship." But despite this relational orientation, members acknowledge that one should tend to one's needs first: "I decided to live life for my own happiness"; "you have to put yourself first here"; "What would make you happy? Not anyone else, but you?"; "Don't worry about anyone else but you."

Ultimately, SF users discursively envision a self that possesses agency and self-determination. In one particular thread, the topic creator (TC) writes about feeling coerced by his father to join the military and turns to other SF users for advice. The responses are rather telling, to say the least.

You're the only one who has a right to decide though, since you are the only one who has to live your life.

Fact is, you don't have to do anything you don't want to in this world.
I think you have every right to live as you please. Sometimes it takes a bit of time to create this life, so patience is important, but once you are independent you have the rest of your life to live however you like.

In short, we are the masters of our own fates, and thus, should be free from imposition. One's life is one's own to live. This cultural proposition has fascinating parallels to an earlier proposition that suicide is enacted by the self, for the self (see Flanigan and Alvarez, 2019). Just as death is one's own, so too is life.

\section{DISCUSSION}

In line with; Burke 1963-1964 proposition that human beings are goaded by the spirit of hierarchy and "rotten with perfection" (p. 509), every discursive community, including SuicideForum.com, subscribes to an ideal of what it means to be a person. This symbolic ghost, which Burke derives from the Aristotelian concept of entelechy (p. 507), haunts individuals when they fail to realize such an ideal. In tracking SF members' usage of discursive hubs like "suicide" and "suicidal" in their online communication, two overarching discursive themes regarding personhood emerge, one relating to problematic ways of being, the other to a self liberated from suicidality.

From SF members' discursive point of view, an individual has a self, and this self can be fragmented or bifurcated. The self can be split across multiple axes - for instance, between an authentic yet problematic inner self, and a socially aligned yet inauthentic outer self. The split can also occur temporally, between past and present, present and future, actual versus ideal(ized) selves. Bifurcation of the self can produce much anguish and may eventuate in thoughts and feelings of suicide. Though a split self is not necessarily problematic in many cultural milieus, it is a source of undue suffering for members of SF, and the sundered parts demand reconciliation (though not necessarily integration).

No one deserves to be suicidal, but anyone can become so. Suicide does not discriminate on the basis of identity markers such as class, gender identity, sexual orientation, faith, or age; the specter of suicide looms even in childhood. The suicidal person is ambivalent and oscillates between life and death, their sense of purpose evacuated of meaning. However, for the pendulum to be on the side of life, s/he must regain purpose and establish meaningful ties to others. No person can exist inside a vacuum, and staying alive - continuing to $b e$ - is (to borrow the words of Heidegger [1927/2010]) contingent upon beingwith-others.

As we have seen, SF users' online communication also invokes notions of a self emancipated from suicidality. According to users' folk understanding, every life, including one's own, has value. No one deserves to be suicidal, and everyone deserves a chance to feel good about themselves. People are also more than their biological makeup. They must tend to the needs of both body and mind, and deal with emotional pain in their own way, in their own time. Furthermore, one's life is one's own to live. Everyone is 
accountable for their own actions, and before tending to others, they must tend to their own needs first.

In the struggle against suicide, willpower alone is not enough. To continue living, one must live meaningfully, for which a sense of purpose is necessary. Purpose can be derived from many sources, including one's passions, aspirations for the future, and suffering, whose meaning is for the suicidal person to discover. One does not have a monopoly on suffering, for everyone experiences hardships, and a person who overcomes suicide is capable of overcoming any adversity. Though each person's struggle in life is unique, we are all connected by our shared humanity. Finally, in spite of all the hardships, one must remain good and strive to always change for the better.

To summarize, the discourses of SF users presume for its formulation an ideal or model person, one that possesses agency over the trajectory of his or her life, inner strength and fortitude, a sense of purpose, and the capacity to find meaning in suffering. The ideal self that emerges in discourse, in the wake of a suicidal crisis, is not necessarily one that is whole, but one that is able to navigate its contradictory parts, without forsaking one's responsibility to contribute to others' well-being. If a goal of therapy or counseling is to help patients, clients, and survivors to actualize the model person they aspire to be, then it stands to reason that existing treatment regimens can benefit from incorporating suicidal persons' own terms and meanings. As Hornstein (2009) has argued, patient-led peer support groups that respect the experiential knowledge and inner wisdom of the so-called mentally ill, such as Hearing Voices and Alternatives to Suicide, have much to teach professionals. At present, in the realm of suicidality, the scholarly literature on offline peer-to-peer (P2P) support is heavily biased towards people bereaved by suicide. Literature on offline P2P support among individuals struggling with thoughts of suicide, outside clinical contexts, is lacking, making comparisons between online and offline prorecovery venues difficult to draw. Nevertheless, SF members' grounded discourses are not without implications for clinical and therapeutic practice that take place offline.

First, realizing that a suicidal patient may be agonizing over a fractured sense of self, will allow the clinician to incorporate strategies for the management and alignment of spoiled identity. Second, the clinician ought to commend the fortitude it takes for a suicidal person to remain alive - a sharp contrast to organized psychiatry's emphasis on deficits, on deviations from established (and often unrealistic) thresholds of normalcy (Reznek, 2016). Instead of treating the suicidal person as defective, s/he will recognize their resilience and respect their right to selfdetermination. Recognizing the agency of suicidal persons does not mean approving the choice to end a life. Rather, it

\section{REFERENCES}

Alvarez, M. (2020). "Discursive Tensions and Contradictions: A Cultural Analysis of an Online Self-Harm Forum," in Communicating with, about, and through Self-Harm: Scarred Discourse. Editor W. Bareiss (Lanham, MD: Lexington Books), 113-133. means enlisting suicidal persons as active participants in their own recovery, rather than passive recipients of paternalistic care. In other words, suicidal persons' claims to agency over the termination of their life (see Flanigan and Alvarez, 2019), rather than contradicted, should be channeled towards the resumption of life.

Third, given the supportive orientation exhibited by SF users in discourse, clinicians need to emphasize to their suicidal clients the social and communal good of staying alive. A similar argument has been made by Hecht (2014), who points out that in choosing to live, the suicidal person benefits his or her immediate community in some way, however small. To this I would add that the suicidal person benefits as well. Reciprocal acts are circular, such that in helping others, one is also helping oneself. In recognizing that suicidal persons are still capable of having a positive impact on the lives around them, their agency is also respected.

Lastly, recovery does not end with the administration of treatment and subsequent amelioration of symptoms. Vocational and rehabilitative efforts cannot afford to ignore the existential dimensions of suicidality - particularly, the salvific role of purpose and meaning. It is not enough for suicidal thoughts and feelings to be attenuated; they must also be replaced with a "zest for life," to borrow the words of one user. Should clinicians listen to patients' stories for their deep meanings, for their beats and rhythms - as opposed to listening for symptoms the way a physician listens for heart murmurs - the lifeworlds of suicidal persons open up and become humanly and discursively intelligible.

\section{DATA AVAILABILITY STATEMENT}

This study analyzed a publicly accessible and anonymous online forum.

\section{AUTHOR CONTRIBUTIONS}

MA is solely responsible for the conception and design of the study, for the collection and analyses of data, and for writing the manuscript in its entirety, submitted here with his approval.

\section{ACKNOWLEDGMENTS}

The author would like to thank Donal Carbaugh, Martin Norden, Daphne Patai, and Jarice Hanson for their comments on the dissertation project from which this article was drawn. I would also like to thank the two reviewers for their detailed feedback on the article. Any errors that remain are my own.

Baker, D., and Fortune, S. (2008). Understanding Self-Harm and Suicide Websites. Crisis 29 (3), 118-122. doi:10.1027/0227-5910.29.3.118

Baume, P., Cantor, C. H., and Rolfe, A. (1997). Cybersuicide: The Role of Interactive Suicide Notes on the Internet. Crisis 18 (2), 73-79. doi:10.1027/0227-5910.18.2.73

Baym, N., and Markham, A. (2009). "Introduction: Making Smart Choices on Shifting Ground," in Internet Inquiry: Conversations about Method. Editors A. Markham and N. Baym (Thousand Oaks, CA: SAGE), vii-xix. 
Biddle, L., Derges, J., Mars, B., Heron, J., Donovan, J. L., Potokar, J., et al. (2016). Suicide and the Internet: Changes in the Accessibility of Suicide-Related Information between 2007 and 2014. J. Affective Disord. 190, 370-375. doi:10.1016/j.jad.2015.10.028

Burke, K. (1963-1964). Definition of Man. Hudson Rev. 16 (4), 491-514. doi:10. $2307 / 3848123$

Carbaugh, D. (2012). "A Communication Theory of Culture," in Inter/cultural Communication: Representation and Construction of Culture. Editor A. Kurylo (Thousand Oaks, CA: SAGE), 69-87.

Carbaugh, D. (2007). Cultural Discourse Analysis: Communication Practices and Intercultural Encounters. J. Intercultural Commun. Res. 36 (3), 167-182. doi:10. 1080/17475750701737090

Carbaugh, D. (2005). Cultures in Conversation. New York, NY: Lawrence Erlbaum.

Carbaugh, D. (2019). "Epilogue," in Engaging and Transforming Global Communication through Cultural Discourse Analysis: A Tribute to Donal Carbaugh. Editors M. Scollo and T. Milburn (Madison, NJ: Fairleigh Dickinson University Press), 313-322.

Carbaugh, D., and Hastings, S. O. (1992). A Role for Communication Theory in Ethnography and Cultural Analysis. Commun. Theor. 2, 156-165. doi:10.1111/j. 1468-2885.1992.tb00035.x

Carbaugh, D. (2017). “Terms for Talk, Take 2: Theorizing Communication through its Cultural Terms and Practices," in The Handbook of Communication in CrossCultural Perspective. Editor D. Carbaugh (New York, NY: Routledge), 15-28.

Chang, S.-S., Page, A., and Gunnell, D. (2011). Internet Searches for a Specific Suicide Method Follow its High-Profile Media Coverage. Ajp 168 (8), 855-857. doi:10.1176/appi.ajp.2011.11020284

E. Aboujaoude and V. Starcevic (2015). in Mental Health in the Digital Age: Grave Dangers, Great Promise (New York, NY: Oxford University Press).

Ekman, I., and Söderberg, S. (2009). "Across the Street - Not Down the Road" Staying Alive through Deliberate Self-Harm," in Internet and Suicide. Editors L. Sher and A. Vilens (Hauppauge, NY: Nova Science Publishers), 221-232.

Flanigan, J., and Alvarez, M. (2019). "Cultural Discourse Analysis as Critical Analysis: A View from Twin Oaks and SuicideForum.Com," in Engaging and Transforming Global Communication through Cultural Discourse Analysis: A Tribute to Donal Carbaugh. Editors M. Scollo and T. Milburn (Lanham, MD: Fairleigh Dickinson University Press), 73-90.

Geertz, C. (1973). The Interpretation of Cultures: Selected Essays. New York, NY: Basic. Giles, D. C., and Newbold, J. (2013). 'Is This normal?' the Role of Category Predicates in Constructing Mental Illness Online. J. Comput-mediat Comm. 18, 476-490. doi:10.1111/jcc4.12022

Goffman, E. (1963). Stigma: Notes on the Management of Spoiled Identity. New York, NY: Simon \& Schuster.

Goffman, E. (1959). The Presentation of Self in Everyday Life. New York, NY: Anchor.

Gunnell, D., Derges, J., Chang, S.-S., and Biddle, L. (2015). Searching for Suicide Methods. Crisis 36 (5), 325-331. doi:10.1027/0227-5910/a000326

Hecht, J. M. (2014). Stay: A History of Suicide and the Arguments against it. New Haven, CT: Yale University Press.

Hecht, M. (2010). "The Promise of Communication in Large-Scale, CommunityBased Research," in Distinctive Qualities in Communication Research. Editors D. Carbaugh and P. M. Buzzanell (New York, NY: Routledge), 53-72.

Heidegger, M. (1927/2010). Being and Time. Albany, NY: SUNY Press.

Hornstein, G. (2009). Agnes's Jacket: A Psychologist's Search for the Meaning of Madness. Emmaus, PA: Rodale. doi:10.36019/9780813549095

Hymes, D. (1972). "Models of Interaction of Language and Social Life," in Directions in Sociolinguistics: The Ethnography of Communication. Editors J. Gumperz and D. Hymes (New York, NY: Holt Rinehart \& Winston), 35-71.

Ikunaga, A., Nath, S. R., and Skinner, K. A. (2013). Internet Suicide in Japan: A Qualitative Content Analysis of a Suicide Bulletin Board. Transcult Psychiatry 50 (2), 280-302. doi:10.1177/1363461513487308

Joiner, T. (2005). Why People Die by Suicide. Cambridge, MA: Harvard University Press.

Katz, J. E., and Rice, R. E. (2002). Social Consequences of Internet Use: Access, Involvement, and Interaction. Cambridge, MA: MIT Press. doi:10.7551/mitpress/6292.001.0001

Kotani, M. (2002). Expressing Gratitude and Indebtedness: Japanese Speakers' Use of "I’m Sorry" in English Conversation. Res. Lang. Soc. Interaction 35, 39-72. doi:10.1207/s15327973rlsi35-1_2

L. Sher and A. Vilens (2009). in Internet and Suicide (Hauppauge, NY: Nova Science Publishers).

Markham, A. (1998). Life Online: Researching Real Experience in Virtual Space. Walnut Creek, CA: AltaMira Press.
Mars, B., Heron, J., Biddle, L., Donovan, J. L., Holley, R., Piper, M., et al. (2015). Exposure to, and Searching for, Information about Suicide and Self-Harm on the Internet: Prevalence and Predictors in a Population Based Cohort of Young Adults. J. Affective Disord. 185, 239-245. doi:10.1016/j.jad.2015.06.001

Mewton, L., and Andrews, G. (2015). Cognitive Behaviour Therapy via the Internet for Depression: A Useful Strategy to Reduce Suicidal Ideation. J. Affective Disord. 170, 78-84. doi:10.1016/j.jad.2014.08.038

M. Scollo and T. Milburn (2019). in Engaging and Transforming Global Communication through Cultural Discourse Analysis: A Tribute to Donal Carbaugh (Madison, NJ: Fairleigh Dickinson University Press).

Ozawa-de Silva, C. (2010). Shared Death: Self, Sociality and Internet Group Suicide in Japan. Transcult Psychiatry 47 (3), 392-418. doi:10.1177/1363461510370239

Ozawa-de Silva, C. (2008). Too Lonely to Die Alone: Internet Suicide Pacts and Existential Suffering in Japan. Cult. Med. Psychiatry 32, 516-551. doi:10.1007/ s11013-008-9108-0

Philipsen, G. (1997). "A Theory of Speech Codes," in Developing Communication Theories. Editors G. Philipsen and T. Albrecht (Albany, NY: SUNY Press), 119-156.

Philipsen, G. (1987). "The prospect for Cultural Communication," in Communication Theory: Eastern and Western Perspectives. Editor D. L. Kincaid (Thousand Oaks, CA: SAGE), 245-254. doi:10.1016/b978-0-12-407470-5.50025-6

Quinnett, P., and Baker, A. (2009). "Web-based Suicide Prevention Education: Innovations in Research, Training, and Practice," in Internet and Suicide. Editors L. Sher and A. Vilens (Hauppauge, NY: Nova Science Publishers), 41-61.

Rajagopal, S. (2004). Suicide Pacts and the Internet. Bmj 329, 1298-1299. doi:10. 1136/bmj.329.7478.1298

Recupero, P. (2012). Suicide and the Internet, in Textbook of Suicide Assessment and Management. Editors R. I. Simon and R. E. Hales. 2nd ed. (Arlington, VA: American Psychiatric Publishing), 515-538.

Reznek, L. (2016). Peddling Mental Disorder: The Crisis in Modern Psychiatry. Jefferson, NC: McFarland.

Scollo, M. (2011). Cultural Approaches to Discourse Analysis: A Theoretical and Methodological Conversation with Special Focus on Donal Carbaugh's Cultural Discourse Theory. J. Multicultural Discourses 6 (1), 1-32. doi:10.1080/ 17447143.2010 .536550

Seko, Y. (2018). "On Deathcasting: Alone Together on the Edge of Death," in Digital Media Usage Across the Lifecourse. Editors P. G. Nixon, R. Rawal, and A. Funk (Abingdon, UK: Routledge), 172-187.

Seko, Y. (2008). "Suicide Machine" Seekers: Transgressing Suicidal Taboos Online. Learn. Inq. 2, 181-199. doi:10.1007/s11519-008-0035-3

Singaravelu, V., Stewart, A., Adams, J., Simkin, S., and Hawton, K. (2015). Information-Seeking on the Internet. Crisis 36 (3), 211-219. doi:10.1027/ 0227-5910/a000307

Stamenkovic, M. (2011). In Full View: Cybersuicide, Hypervisual Self and Public Showcase of Death (For Real). Medijski Dijalozi 5 (12), 73-101. http://hdl. handle.net/1854/LU-4443210

Stern, S. R. (2003). Encountering Distressing Information in Online Research: A Consideration of Legal and Ethical Responsibilities. New Media Soc. 5, 249-256. doi:10.1177/1461444803005002006

SuicideForum.com (n.d.). About SF. Retrieved September 30, 2016, from Available at: https://www.suicideforum.com/about-sf/.

Thompson, R. (2012). Screwed up, but Working on it. Ni 22 (1), 86-104. doi:10. 1075/ni.22.1.06tho

Westerlund, M. (2013). Talking Suicide. Nordicom Rev. 34, 35-46. doi:10.2478/ nor-2013-0052

Westerlund, M. (2011). The Production of Pro-suicide Content on the Internet: A Counter-discourse Activity. New Media Soc. 14, 764-780. doi:10.1177/ 1461444811425221

Conflict of Interest: The author declares that the research was conducted in the absence of any commercial or financial relationships that could be construed as a potential conflict of interest.

Copyright $\odot 2021$ Alvarez. This is an open-access article distributed under the terms of the Creative Commons Attribution License (CC BY). The use, distribution or reproduction in other forums is permitted, provided the original author(s) and the copyright owner(s) are credited and that the original publication in this journal is cited, in accordance with accepted academic practice. No use, distribution or reproduction is permitted which does not comply with these terms. 